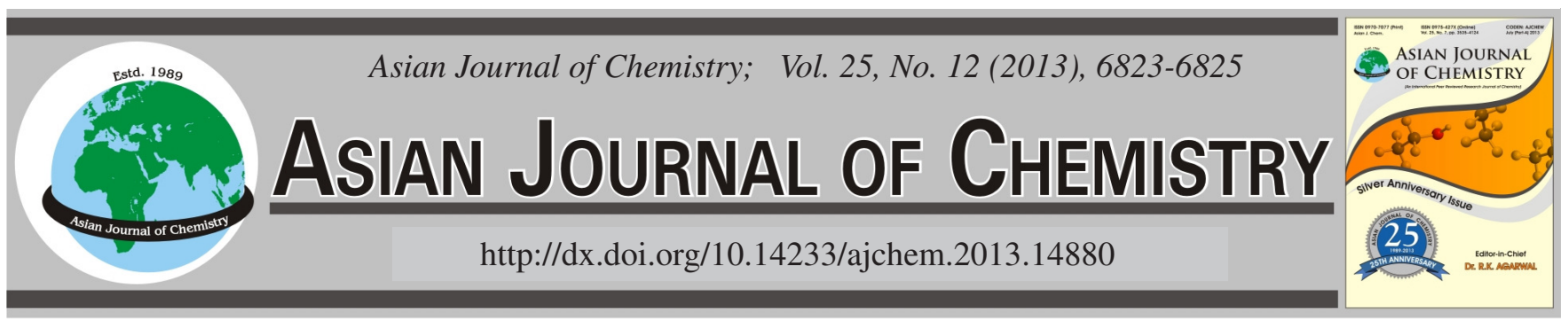

\title{
A New Acentric Factor Correlation for Pure Hydrocarbons
}

\author{
Ali Akbar Amooey*, Smaneh Heydari and Mahbobeh Ahmadi
}

Department of Chemical Engineering, University of Mazandaran, Babolsar, Iran

*Corresponding author: Tel: +98 1125342903; E-mail: aamooey@umz.ac.ir

(Received: 26 November 2012;

Accepted: 5 June 2013)

AJC-13580

In this study, a vapour pressure equation is proposed to correlate saturated vapour pressure of pure compounds with higher accuracy. The acentric factor of 57 pure hydrocarbons is estimated based on this proposed equation for pure hydrocarbons. The results show that proposed equation is better than modified Wagner equation for estimating the acentric factor of pure hydrocarbons.

Key Words: Critical properties, Hydrocarbon, Vapour pressure, Acentric factor, Correlation.

\section{INTRODUCTION}

The acentric factor values are essential in estimating transport and thermodynamic properties for liquids and gases such as enthalpy of vapourization ${ }^{1}$, saturated density or molar volume of liquids ${ }^{2,3}$, compressibility factor ${ }^{4-7}$, heat capacity of real gas ${ }^{7}$, vapour pressure of pure liquid ${ }^{7}$ and saturated liquid viscosity $^{8,9}$. The acentric factor represents the acentricity or non- sphericity of a molecule.

In this work, a vapour pressure equation is suggested to correlate saturated vapour pressure of pure compounds and then the acentric factor is estimated according to the proposed equation for pure hydrocarbons and compared with modified Wagner. The average absolute error of proposed equation is 0.0507 while for modified Wagner's equation is 0.0847 . The results show that the equation has good accuracy for estimating the acentric factor of pure hydrocarbon.

\section{Proposed equation}

The new saturated vapour pressure equation is proposed as follow:

$$
\begin{gathered}
\ln \mathrm{P}_{\mathrm{r}}^{\mathrm{s}}=\left[\left(\mathrm{a}+\mathrm{bt}+\mathrm{ct}^{5}+\mathrm{d} \exp (\mathrm{e}(1-\mathrm{t}))\right) \log (\mathrm{t})\right] / \mathrm{t} \\
\mathrm{t}=\frac{\mathrm{T}}{\mathrm{T}_{\mathrm{c}}} \\
\operatorname{Pr}^{\mathrm{s}}=\frac{\mathrm{P}}{\mathrm{P}_{\mathrm{c}}}
\end{gathered}
$$

In this equation, $P_{c}$ is the critical pressure, $T_{c}$ is the critical temperature and a, b, c, d, e are constants.

According to the corresponding state principle, the saturated vapour pressure equation is defined as:

$$
\ln \mathrm{P}_{\mathrm{r}}^{\mathrm{s}}=\mathrm{f}^{(0)}(\mathrm{t})+\omega \mathrm{f}^{(1)}(\mathrm{t})
$$

In the above equation, $\mathrm{f}^{(0)}(\mathrm{t})$ and $\mathrm{f}^{(1)}(\mathrm{t})$ are presented as below, based on the new equation:

$$
\begin{aligned}
& \mathrm{f}^{(0)}=\left[\left(\mathrm{a}_{1}+\mathrm{b}_{1} \mathrm{t}+\mathrm{c}_{1} \mathrm{t}^{5}+\mathrm{d}_{1} \exp \left(\mathrm{e}_{1}(1-\mathrm{t})\right) \log (\mathrm{t})\right] / \mathrm{t}\right. \\
& \mathrm{f}^{(1)}=\left[\left(\mathrm{a}_{2}+\mathrm{b}_{2} \mathrm{t}+\mathrm{c}_{2} \mathrm{t}^{5}+\mathrm{d}_{2} \exp \left(\mathrm{e}_{2}(1-\mathrm{t})\right) \log (\mathrm{t})\right] / \mathrm{t}\right.
\end{aligned}
$$

The constants in eqns. 5 and 6 are calculated by minimization of the objective function:

$$
\mathrm{ADD}=\sum_{\mathrm{i}}^{\mathrm{N}}\left|\frac{\mathrm{P}^{\exp }-\mathrm{P}^{\text {model }}}{\mathrm{P}^{\exp }}\right|
$$

where $\mathrm{p}^{\exp }$ is the experimental pressure, $\mathrm{p}^{\text {model }}$ is calculated by eqns. 4-6 and $\mathrm{N}$ is the number of the experimental data point.

The experimental saturated vapour pressure data for 30 hydrocarbons are obtained by Perry and Green ${ }^{10}$. The constant parameters (a, b, c, d and e) of eqns. 5 and 6 are shown in Table-1. These constant parameters are calculated by regression between experimental data and calculated data.

\begin{tabular}{cccccc}
\multicolumn{7}{c}{ TABLE-1 } \\
\multicolumn{7}{c}{ CONSTANT PARAMETERS OF EQNS. 5 AND 6 } \\
\hline Constant & $\mathrm{a}_{\mathrm{i}}$ & $\mathrm{b}_{\mathrm{i}}$ & $\mathrm{c}_{\mathrm{i}}$ & $\mathrm{d}_{\mathrm{i}}$ & $\mathrm{e}_{\mathrm{i}}$ \\
\hline $\mathrm{i}=1$ & -0.0389 & 4.3098 & 1.2712 & 1.0045 & 1.0650 \\
$\mathrm{i}=2$ & 2.4240 & 1.4458 & -2.7831 & 1.1696 & 0.8115 \\
\hline
\end{tabular}

The acentric factor $(\omega)$ of a pure substance is determined by using the Pitzer equation:

$$
\omega=\left(-\log \left(\mathrm{P}^{\mathrm{s}} / \mathrm{P}_{\mathrm{c}}\right)\right)_{(\mathrm{T} / \mathrm{Tc})=0.7}-1
$$


PROPERTIES OF COMPOUNDS AND THE RESULTS OF THE PROPOSED MODEL, MODIFIED WAGNER's MODEL FOR ESTIMATING ACENTRIC FACTOR

\begin{tabular}{|c|c|c|c|c|c|c|c|c|c|}
\hline \multicolumn{6}{|c|}{ Properties of compounds } & \multicolumn{2}{|c|}{ Modified Wagner } & \multicolumn{2}{|c|}{ Proposed model } \\
\hline Formula & m.w. & $\mathrm{T}_{\mathrm{b}}(\mathrm{K})$ & $\mathrm{T}_{\mathrm{c}}(\mathrm{K})$ & $\mathrm{P}_{\mathrm{c}}$ (bar) & $\omega$ & $\omega_{\text {cal }}$ & AAD & $\omega_{\mathrm{cal}}$ & $\mathrm{AAD}$ \\
\hline $\mathrm{CF}_{4}$ & 88.005 & 145.11 & 227.51 & 37.45 & 0.177 & 0.1769 & 0.0005 & 0.1728 & 0.0239 \\
\hline $\mathrm{CClF}_{3}$ & 104.459 & 191.71 & 301.84 & 38.73 & 0.175 & 0.1737 & 0.0072 & 0.1700 & 0.0284 \\
\hline $\mathrm{CCl}_{2} \mathrm{~F}_{2}$ & 120.913 & 243.45 & 385.10 & 41.30 & 0.179 & 0.1782 & 0.0044 & 0.1750 & 0.0223 \\
\hline $\mathrm{CCl}_{3} \mathrm{~F}$ & 137.368 & 296.81 & 471.10 & 44.72 & 0.195 & 0.1910 & 0.0208 & 0.1882 & 0.0349 \\
\hline $\mathrm{CHClF}_{2}$ & 86.468 & 232.14 & 369.28 & 49.86 & 0.221 & 0.2155 & 0.0251 & 0.2131 & 0.0357 \\
\hline $\mathrm{CHCl}_{2} \mathrm{~F}$ & 102.923 & 281.97 & 451.52 & 51.87 & 0.207 & 0.2058 & 0.0058 & 0.2040 & 0.0144 \\
\hline $\mathrm{CH}_{2} \mathrm{O}_{2}$ & 46.026 & 374.04 & 588.00 & 58.07 & 0.316 & 0.3011 & 0.0473 & 0.2979 & 0.0572 \\
\hline $\mathrm{CH}_{3} \mathrm{~F}$ & 34.033 & 194.88 & 315.00 & 55.48 & 0.204 & 0.1957 & 0.0409 & 0.1946 & 0.0461 \\
\hline $\mathrm{CH}_{4} \mathrm{O}$ & 32.042 & 337.69 & 512.64 & 80.97 & 0.565 & 0.5585 & 0.0115 & 0.5489 & 0.0285 \\
\hline $\mathrm{CO}$ & 28.010 & 81.66 & 132.85 & 34.94 & 0.045 & 0.0528 & 0.1744 & 0.0505 & 0.1231 \\
\hline $\mathrm{C}_{2} \mathrm{Cl}_{2} \mathrm{~F}_{4}$ & 170.921 & 276.59 & 418.70 & 32.13 & 0.244 & 0.2492 & 0.0213 & 0.2401 & 0.0158 \\
\hline $\mathrm{C}_{2} \mathrm{Cl}_{3} \mathrm{~F}_{3}$ & 187.375 & 320.74 & 487.40 & 33.78 & 0.249 & 0.2520 & 0.0122 & 0.2436 & 0.0215 \\
\hline $\mathrm{C}_{2} \mathrm{H}_{2}$ & 26.038 & 188.40 & 308.30 & 61.14 & 0.189 & 0.1861 & 0.0154 & 0.1858 & 0.0168 \\
\hline $\mathrm{C}_{2} \mathrm{H}_{4} \mathrm{~F}_{2}$ & 66.051 & 249.10 & 386.41 & 45.16 & 0.276 & 0.2716 & 0.0158 & 0.2665 & 0.0344 \\
\hline $\mathrm{C}_{2} \mathrm{H}_{4} \mathrm{O}_{2}$ & 60.053 & 391.04 & 594.45 & 57.90 & 0.445 & 0.4361 & 0.0199 & 0.4272 & 0.0399 \\
\hline $\mathrm{C}_{2} \mathrm{H}_{5} \mathrm{~F}$ & 48.060 & 235.43 & 375.28 & 50.27 & 0.217 & 0.2113 & 0.0264 & 0.2091 & 0.0364 \\
\hline $\mathrm{C}_{2} \mathrm{H}_{6}$ & 30.070 & 184.55 & 305.32 & 48.70 & 0.099 & 0.0969 & 0.0215 & 0.0960 & 0.0299 \\
\hline $\mathrm{C}_{2} \mathrm{H}_{6} \mathrm{O}$ & 46.069 & 351.80 & 513.92 & 61.48 & 0.649 & 0.6688 & 0.0982 & 0.6452 & 0.0594 \\
\hline $\mathrm{C}_{2} \mathrm{H}_{7} \mathrm{~N}$ & 45.084 & 289.75 & 456.40 & 56.30 & 0.276 & 0.2846 & 0.0312 & 0.2816 & 0.0204 \\
\hline $\mathrm{C}_{3} \mathrm{H}_{4}$ & 40.065 & 238.77 & 394.00 & 52.50 & 0.122 & 0.1226 & 0.0053 & 0.1221 & 0.0004 \\
\hline $\mathrm{C}_{3} \mathrm{H}_{6} \mathrm{Cl}_{2}$ & 112.187 & 369.43 & 578.00 & 46.50 & 0.255 & 0.2502 & 0.0187 & 0.2462 & 0.0345 \\
\hline $\mathrm{C}_{3} \mathrm{H}_{6} \mathrm{O}$ & 58.080 & 329.22 & 508.10 & 47.00 & 0.307 & 0.3035 & 0.0115 & 0.2976 & 0.0306 \\
\hline $\mathrm{C}_{3} \mathrm{H}_{6} \mathrm{O}_{3}$ & 90.084 & 363.24 & 557.00 & 48.00 & 0.33 & 0.3357 & 0.0010 & 0.3287 & 0.0217 \\
\hline $\mathrm{C}_{3} \mathrm{H}_{8}$ & 44.097 & 231.02 & 369.83 & 42.48 & 0.152 & 0.1502 & 0.0120 & 0.1478 & 0.0274 \\
\hline $\mathrm{C}_{4} \mathrm{H}_{6} \mathrm{O}_{3}$ & 102.090 & 412.69 & 606.00 & 40.00 & 0.456 & 0.9537 & 1.0916 & 0.8811 & 0.9323 \\
\hline $\mathrm{C}_{4} \mathrm{H}_{8} \mathrm{O}$ & 72.107 & 352.71 & 536.80 & 42.10 & 0.322 & 0.3217 & 0.0008 & 0.3134 & 0.0296 \\
\hline $\mathrm{C}_{4} \mathrm{H}_{10} \mathrm{~S}$ & 90.189 & 365.25 & 557.00 & 39.60 & 0.295 & 0.2929 & 0.0072 & 0.2850 & 0.0339 \\
\hline $\mathrm{C}_{5} \mathrm{H}_{5} \mathrm{~N}$ & 79.101 & 388.37 & 620.00 & 56.70 & 0.242 & 0.2409 & 0.0046 & 0.2391 & 0.0118 \\
\hline $\mathrm{C}_{5} \mathrm{H}_{8} \mathrm{O}$ & 84.118 & 403.72 & 624.50 & 46.00 & 0.288 & 0.2879 & 0.0003 & 0.2824 & 0.0194 \\
\hline $\mathrm{C}_{6} \mathrm{H}_{5} \mathrm{Cl}$ & 112.558 & 404.91 & 632.40 & 45.20 & 0.251 & 0.2476 & 0.0136 & 0.2433 & 0.0306 \\
\hline $\mathrm{C}_{6} \mathrm{H}_{6}$ & 78.114 & 353.24 & 562.05 & 48.95 & 0.21 & 0.2094 & 0.0029 & 0.2070 & 0.0142 \\
\hline $\mathrm{C}_{6} \mathrm{H}_{6} \mathrm{O}$ & 94.113 & 455.04 & 694.25 & 61.30 & 0.442 & 0.4393 & 0.0061 & 0.4312 & 0.0244 \\
\hline $\mathrm{C}_{6} \mathrm{H}_{10} \mathrm{O}$ & 98.144 & 428.59 & 653.00 & 40.00 & 0.299 & 0.2999 & 0.0029 & 0.2918 & 0.0241 \\
\hline $\mathrm{C}_{7} \mathrm{H}_{8}$ & 92.141 & 383.79 & 591.75 & 41.08 & 0.264 & 0.2643 & 0.0024 & 0.2573 & 0.0254 \\
\hline $\mathrm{C}_{8} \mathrm{H}_{8} \mathrm{O}$ & 120.153 & 475.26 & 713.00 & 40.30 & 0.361 & 0.3670 & 0.0166 & 0.3554 & 0.0154 \\
\hline $\mathrm{C}_{8} \mathrm{H}_{16} \mathrm{O}_{2}$ & 112.215 & 512.01 & 695.00 & 26.40 & 0.734 & 0.7904 & 0.0769 & 0.7196 & 0.0196 \\
\hline $\mathrm{C}_{8} \mathrm{H}_{19} \mathrm{~N}$ & 129.246 & 451.70 & 641.00 & 26.17 & 0.446 & 0.4699 & 0.0536 & 0.4390 & 0.0158 \\
\hline $\mathrm{C}_{9} \mathrm{H}_{20} \mathrm{O}$ & 144.257 & 486.52 & 668.90 & 26.30 & 0.633 & 0.6838 & 0.0802 & 0.6278 & 0.0082 \\
\hline $\mathrm{C}_{10} \mathrm{H}_{20} \mathrm{O}_{2}$ & 172.268 & 541.92 & 726.00 & 22.30 & 0.749 & 0.8049 & 0.0746 & 0.7223 & 0.0356 \\
\hline $\mathrm{C}_{10} \mathrm{H}_{22} \mathrm{O}$ & 158.284 & 504.25 & 684.40 & 23.70 & 0.661 & 0.7276 & 0.1008 & 0.6603 & 0.0010 \\
\hline $\mathrm{C}_{11} \mathrm{H}_{24}$ & 156.312 & 469.08 & 639.00 & 19.80 & 0.537 & 0.5937 & 0.1056 & 0.5364 & 0.0012 \\
\hline $\mathrm{C}_{11} \mathrm{H}_{24} \mathrm{O}$ & 172.311 & 521.24 & 705.00 & 22.40 & 0.656 & 0.7232 & 0.1024 & 0.6535 & 0.0038 \\
\hline $\mathrm{C}_{12} \mathrm{H}_{26}$ & 170.338 & 489.48 & 658.00 & 18.20 & 0.579 & 0.6470 & 0.1175 & 0.5774 & 0.0028 \\
\hline $\mathrm{C}_{12} \mathrm{H}_{26} \mathrm{O}$ & 186.338 & 537.79 & 720.00 & 20.80 & 0.684 & 0.7665 & 0.1206 & 0.6859 & 0.0027 \\
\hline $\mathrm{C}_{13} \mathrm{H}_{12}$ & 168.238 & 537.65 & 760.00 & 27.10 & 0.481 & 0.5095 & 0.5095 & 0.4756 & 0.0112 \\
\hline $\mathrm{C}_{13} \mathrm{H}_{28}$ & 184.365 & 508.63 & 675.00 & 16.80 & 0.618 & 0.7061 & 0.7061 & 0.6221 & 0.0067 \\
\hline $\mathrm{C}_{14} \mathrm{H}_{22}$ & 190.330 & 510.43 & 708.00 & 23.00 & 0.506 & 0.5483 & 0.5483 & 0.5037 & 0.0045 \\
\hline $\mathrm{C}_{14} \mathrm{H}_{30}$ & 198.392 & 526.76 & 693.00 & 15.70 & 0.644 & 0.7406 & 0.7406 & 0.6461 & 0.0033 \\
\hline $\mathrm{C}_{15} \mathrm{H}_{32}$ & 212.419 & 543.83 & 708.00 & 14.80 & 0.685 & 0.8028 & 0.8028 & 0.6926 & 0.0110 \\
\hline $\mathrm{C}_{16} \mathrm{H}_{34} \mathrm{O}$ & 242.446 & 597.53 & 770.00 & 16.10 & 0.818 & 0.9857 & 0.9857 & 0.8462 & 0.0345 \\
\hline $\mathrm{C}_{17} \mathrm{H}_{36}$ & 240.473 & 574.56 & 736.00 & 13.40 & 0.753 & 0.9062 & 0.9062 & 0.7668 & 0.0184 \\
\hline $\mathrm{C}_{18} \mathrm{H}_{38}$ & 254.500 & 588.30 & 747.00 & 12.90 & 0.800 & 0.9849 & 0.9849 & 0.8248 & 0.0233 \\
\hline $\mathrm{C}_{19} \mathrm{H}_{40}$ & 268.527 & 602.34 & 755.00 & 11.60 & 0.845 & 1.0666 & 1.0666 & 0.8761 & 0.0368 \\
\hline $\mathrm{Br}_{2}$ & 159.808 & 331.90 & 584.10 & 103.00 & 0.119 & 0.1185 & 0.1185 & 0.1202 & 0.0098 \\
\hline $\mathrm{HI}$ & 127.912 & 237.57 & 423.90 & 90.00 & 0.038 & 0.0606 & 0.5943 & 0.0614 & 0.6150 \\
\hline $\mathrm{H}_{2} \mathrm{O}$ & 18.015 & 373.15 & 647.14 & 220.64 & 0.344 & 0.3194 & 0.0715 & 0.3243 & 0.0572 \\
\hline NO & 30.006 & 121.38 & 180.00 & 64.80 & 0.582 & 0.6016 & 0.0337 & 0.5846 & 0.0044 \\
\hline Average & & & & & & 0.0847 & & 0.0507 & \\
\hline
\end{tabular}


The saturated vapour pressure at $\mathrm{T} / \mathrm{Tc}=0.7$ is required for estimating the acentric factor.

The acentric factor is calculated by using the values at the normal boiling point:

$$
\text { at } \mathrm{T}=\mathrm{T}_{\mathrm{b}} \quad \Rightarrow \mathrm{P}=1.01325 \mathrm{bar}
$$

Thus, eqn. 4 is changed to:

$$
\omega=-\left(\ln \left(\mathrm{P}_{\mathrm{c}} / 1.01325\right)+\mathrm{f}^{(0)}(\mathrm{v})\right) / \mathrm{f}^{(1)}(\mathrm{v})
$$

where

$$
\begin{gathered}
f^{(0)}(v)=\frac{\left[-0.0389+4.3098 v+1.2712 v^{5}+1.0045 \exp (1.065(1-v))\right] \log (v)}{v}(11) \\
f^{(1)}(v)=\frac{\left[2.424+1.4458 v-2.7831 v^{5}+1.1696 \exp (0.8115(1-v))\right] \log (v)}{v}(12) \\
v=\frac{T_{b}}{T_{c}}
\end{gathered}
$$

where $T_{b}$ is boiling temperature.

\section{RESULTS AND DISCUSSION}

Acentric factor is estimated for 57 pure hydrocarbons by eqn. 10. The normal boiling temperature $\left(\mathrm{T}_{\mathrm{b}}\right.$ for $\mathrm{P}_{\mathrm{b}}=1.01325$ bar), the critical properties, acentric factor and the average absolute deviations of new model and modified Wagner model ${ }^{11}$ are presented in Table-2.

The modified Wagner equation has the following form:

$$
\begin{aligned}
& \ln \mathrm{P}_{\mathrm{r}}^{\mathrm{s}}=\left(-5.462 \mathrm{t}+0.0666 \mathrm{t}^{1.5}+0.9311 \mathrm{t}^{3}-5.8487 \mathrm{t}^{6}\right) /(1-\mathrm{t})+ \\
& \omega\left(1.9892 \mathrm{t}-13.4124 \mathrm{t}^{1.5}+1.9574 \mathrm{t}^{3}+9.2168 \mathrm{t}^{6}\right) /(1-\mathrm{t})
\end{aligned}
$$

$\mathrm{t}=1-\mathrm{T} / \mathrm{T}_{\mathrm{c}}$
According to Table-2, the average absolute deviation of new model for 57 pure hydrocarbons is 0.0507 and for modified Wagner model (eq. 14) is 0.0847 . This result shows that the new model has a better accuracy than modified Wagner model.

\section{Conclusion}

The saturated vapour pressure is calculated for 30 hydrocarbons according to the proposed equation. In addition, acentric factor $(\omega)$ is calculated for 57 pure hydrocarbon based on the corresponding state principle. Based on the average absolute deviation values, the proposed equation has good accuracy for estimating acentric factor.

\section{REFERENCES}

1. J. Nath, Ind. Eng. Chem. Fundam., 8, 297 (1979).

2. H.G. Rackett, J. Chem. Eng. Data, 15, 514 (1970)

3. G.H. Thompson, K.R. Brobst and R.W. Hankinson, AIChE J., 28, 671 (1982).

4. K.S. Pitzer, J. Am. Chem. Soc., 77, 3427 (1955).

5. K.S. Pitzer, D.M. Lippmann, R.F. Curl, C.M. Huggins and D.E. Peterson, J. Am. Chem. Soc., 77, 3433 (1955).

6. W.C. Edmister, Pet. Refin., 37, 173 (1958).

7. B.I. Lee and M.G. Kesler, AIChE J., 21, 510 (1975).

8. A. Letsou and L.I. Stiel, AIChE J., 19, 409 (1973).

9. R.P. Danner and T.E. Daubert, Data Prediction Manual, DIPPR, AIChE: New York (1983).

10. R.H. Perry and D.W. Green, Perry Chemical Engineers Handbook, Tokyo: McGraw Hill (1988).

11. W. Wagner, Cryogenics, 13, 470 (1973). 